Ophthalmologe 2013 · 110:402

DOI 10.1007/s00347-012-2700-2

Online publiziert: 1. März 2013

๑) Springer-Verlag Berlin Heidelberg 2013

\section{H. Wilhelm}

Universitäts-Augenklinik, Universitätsklinikum Tübingen

\section{Meningeome der vorderen Sehbahn}

Chiasma-nahe Tumoren, also solche, die das Sehvermögen beeinträchtigen können, sind Hypophysenadenome, Kraniopharyngeome, Meningeome, Aneurysmen und sehr selten einmal etwas anderes. So habe ich es gelernt, als ich vor 30 Jahren mit der Augenheilkunde anfing. Wenn ich zurückblicke, dann war unser größter Fehler im Hinblick auf diese Tumoren, dass wir sie alle in einen Topf geworfen haben. Hypophysenadenome sind meistens unproblematisch, sie lassen sich gut operieren, transnasal, manchmal sogar konservativ behandeln.

Meningeome sind anders. Sie wachsen langsam, manchmal sehr langsam. Selten kann der Patient den Tag nennen, an dem sein Sehen schlechter wurde. Es dauert sehr lange, bis er merkt, dass etwas nicht mehr stimmt. Damit ähneln Meningeome dem Glaukom, das auch heimlich fortschreitet und mit dem sie gerne verwechselt werden. Sie sind im Gegensatz zum Hypophysentumor nicht zu spektakulären Katastrophen wie einem Tumorapoplex in der Lage, und sie bilden auch keine Zysten wie das Kraniopharyngeom. Aber Meningeomen gelingt es oft heimlich, so groß zu werden, dass sie nicht mehr ohne weiteren Schaden operiert werden können. Wir alle erinnern uns an Patienten, die nach der Operation schlechter sahen als zuvor.

In den letzten Jahren hat sich viel geändert. Die neurochirurgischen Techniken sind besser geworden, die Strahlentherapie ist als ebenbürtiger Partner hinzugekommen. Tumoren, die als inoperabel galten, wurden behandelbar. Die Diagnose wird vom Neuroradiologen vielfach ohne Biopsie gestellt und die Bestrahlung erfolgt ohne sichere Histologie, vor 20 Jahren war das kaum vorstellbar. Aber immer noch gilt die Regel, dass es keine effizien- te Tumortherapie ohne Risiken gibt. Die Indikation zur Behandlung eines Meningeoms ist keinesfalls sein Nachweis in der MRT oder im PET-CT. Entscheidend sind immer die Funktion und die Prognose. Bei gutem Gesichtsfeld und nur gering reduziertem Visus darf man gerne zunächst einmal beobachten, vielleicht stellt der Tumor sein Wachstum von sich aus ein, oder er hat es vielleicht längst eingestellt. Viele Meningeome werden nämlich nicht deshalb gefunden, weil sie Beschwerden verursachen, sondern sind zufälliger „Beifang" bei einem Beruhigungs-MRT wegen Kopfschmerzen oder Schwindel. „Inzidentalome“ nennt man solche Tumoren.

\section{I) Bei Meningeomen der vorderen Sehbahn entscheidet der augenärztliche Befund}

Wir Augenärzte haben in der Vergangenheit nicht genug getan. Wo wir klare Vorgaben hätten machen müssen, haben wir Patienten an neurologische Kollegen verwiesen, in der Vorstellung, diese kämen mit Tumorpatienten besser zurecht. Das mag für die meisten Hirntumoren seine Richtigkeit haben, nicht jedoch für die Meningeome der vorderen Sehbahn. Hier entscheidet der augenärztliche Befund. Ein Tumor mit progredientem Gesichtsfeldausfall und noch einigermaßen vitaler Papille muss behandelt werden, ein Tumor ohne Ausfälle braucht keine Therapie, gleichfalls gibt es bei einem winzigen Gesichtsfeldrest und weißer Papille nichts zu gewinnen. Dies alles ist für den Nicht-Ophthalmologen kaum zu beurteilen. Und so fiel allzu oft die Entscheidung außerhalb der Augenheilkunde: „Im
Zweifelfall lieber operieren!“, was nicht immer die richtige Entscheidung war.

Dieses Themenheft soll den Augenarzt auf den neuesten Stand hinsichtlich Diagnostik und Therapie bringen. Wir sind bewusst als Tübinger „unter uns“ geblieben, denn das $\mathrm{A}$ und $\mathrm{O}$ der Betreuung dieser Patienten ist eine gute Zusammenarbeit der beteiligten Fächer, Augenheilkunde, Neuroradiologie, Neurochirurgie und Radioonkologie. Mit diesem Themenheft stellen wir uns als ein solches Team vor, insbesondere in dem Beitrag, in dem es um konkrete Fälle geht. Da wird auch klar, dass wir nicht immer einer Meinung sind. Man kann es gar nicht sein, zu komplex ist das Thema. Aber voneinander zu wissen, sich zu kennen und Fälle zu diskutieren führt mit großer Sicherheit immer zu einer im Einzelfall optimalen Lösung. Diese Interaktion, in die der Augenarzt seine besonderen Kenntnisse um Funktion und Prognose einbringt, ist entscheidend dafür, dass jeder Patient die angemessene Therapie erhält.
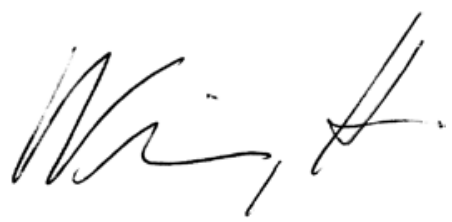

Helmut Wilhelm

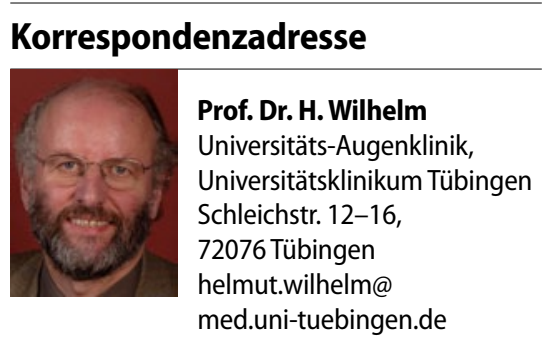

